\title{
Application of a near-wall domain decomposition method to turbulent flows with heat transfer
}

\author{
Adam Jones ${ }^{\mathrm{a}, *}$, Sergey Utyuzhnikov ${ }^{\mathrm{a}, \mathrm{b}}$ \\ ${ }^{a}$ School of Mechanical, Aerospace and Civil Engineering, University of Manchester, Manchester M13 9PL, UK \\ ${ }^{\mathrm{b}}$ Moscow Institute of Physics and Technology, Dolgoprudny 141700, Russia
}

\section{A R T I C L E I N F O}

\section{Article history:}

Received 27 November 2014

Received in revised form 31 March 2015

Accepted 26 June 2015

Available online 3 July 2015

\section{Keywords:}

Domain decomposition

Interface boundary conditions

Robin boundary condition

Wall functions

Turbulence

\begin{abstract}
A B S T R A C T
A near-wall domain decomposition method for use in turbulence modelling is applied to the $k-\omega$ SST, Spalart-Allmaras and BL- $\overline{v^{2}} / k$ turbulence models. The near-wall region is excluded from the main computational mesh. This eliminates the expense of computing the solution in the viscous sub layer and reduces the total computation time.

A one-dimensional boundary layer equation is used to transfer the wall boundary condition from the wall to an interface located within the flow domain. The boundary conditions imposed on the interface are of Robin type and are written in mesh-independent form. The boundary layer equation can contain source terms such as the pressure gradient or near-wall damping terms. Scalar boundary conditions can be calculated using the same formalism as the boundary conditions for the velocity.

The implementation of the boundary conditions is tested on a channel flow, two heated annulus flows and a two-dimensional, asymmetric diffuser. For each case, different locations of the interface boundary are tested. The results are not sensitive to the location of the interface. Friction factors and heat transfer data calculated with the domain decomposition approach compare well to the results obtained with the fully-resolved forms of the respective turbulence models.
\end{abstract}

(c) 2015 Elsevier Ltd. All rights reserved.

\section{Introduction}

Fluid flows in practical engineering applications often have very large Reynolds numbers. In such flows, a boundary layer develops near to the walls in which the velocity of the fluid changes rapidly with distance from the wall. This poses a significant computational challenge. In order to capture the large gradients in the boundary layer, the computational mesh must be very fine near the wall. This leads to a large computation time.

In large eddy simulation (LES), the computational requirement of resolving the near-wall layer often makes a wall-resolved LES computation impractical. However as computing power increases, it is becoming more common to fully resolve the boundary layers in a computational fluid dynamics (CFD) calculation, particularly with Reynolds averaged Navier-Stokes (RANS) models. Turbulence models that resolve the boundary layer are called low Reynolds number (LRN) models. However the time requirements of resolving the near-wall layer can be as much as $90 \%$ of the total computation time. This is unappealing for industrial applications, where a solution is often required in a short space of time.

\footnotetext{
* Corresponding author. Tel.: +44 1613063707.

E-mail addresses: adam.jones-2@manchester.ac.uk (A. Jones),s.utyuzhnikov@ manchester.ac.uk (S. Utyuzhnikov).
}

For these reasons, various approaches have been developed to simplify the modelling of the near-wall regions of flows. In RANS computations, wall functions are often used. These are semi-empirical correlations that link the velocity at the cell nearest to the wall with the shear stress at the wall. This allows a coarse grid to be used near to the wall whilst retaining an adequate approximation of the wall shear stress. The coarser grid reduces the computational cost of the simulation. Models that use wall functions are called high-Reynolds number (HRN) models. The wall function represents the inner (near-wall) region while the turbulence model represents the outer region [16].

The earliest wall functions are based on the log law, which is valid under certain circumstances in the fully turbulent region of the boundary layer. The logarithmic relationship between wall distance and flow velocity is used to calculate the wall shear stress. However the log law is only valid in the logarithmic region of the boundary layer. If the near-wall cell is located in the viscous sub layer then the wall function fails. Furthermore in many flows, such as those with separation regions, the logarithmic region of the boundary layer does not exist at all. Such wall functions are commonly used in engineering applications today, even though they are of limited accuracy, especially if the near-wall cell centre does not lie in the logarithmic region of the boundary layer. 
An improvement to this type of wall function is the scalable wall function (SWF) [18]. With this approach, if the near-wall cell happens to be in the viscous sub layer, boundary conditions are imposed as if the cell were at the very edge of the viscous sub layer. The log law is still used to compute the wall shear stress, however the requirement that the near-wall cell centre lie in the logarithmic region of the boundary layer has been removed. For this reason, and also because of its simplicity, the SWF is a commonly used wall function. However the wall function cannot account for pressure gradients, and is invalid in flows where separation occurs.

In recent years, more sophisticated wall functions have been developed to try to further improve the range of validity of wall functions. One such example is the analytical wall function [12]. This wall function assumes a piecewise linear variation of the turbulent viscosity near to the wall, which permits analytical integration of the momentum boundary layer equations across the boundary layer. This wall function does not explicitly use the log law. In addition, the pressure gradient is included in the integration, which improves the accuracy of the boundary conditions compared to log law-based approaches. This wall function is not widely used in industrial CFD codes because the analytical expressions are rather long and cumbersome, especially since special treatment is required if the near-wall cell centre lies in the viscous sublayer. Moreover it is difficult to generalise this approach to unstructured codes.

Another example of an improved wall function is the numerical wall function (NWF) [11], which does not assume a viscosity profile. A one-dimensional numerical grid is used to solve a simplified version of the momentum equations in every near wall cell. This means that the NWF is, in general, more accurate than the AWF. However, this wall function suffers from stability issues [17]. Implementation of this approach also requires significant modifications to the underlying CFD code, and is fraught with technical issues in unstructured codes.

The alternative to using wall functions is to use a near-wall domain decomposition (NDD) method [36]. With NDD approaches, the computational domain is split into an outer region and the inner region, which is near to the wall. In contrast to wall functions, which are intrinsically mesh-dependent [16], the NDD approach seamlessly merges the solution in the inner and outer regions via mesh-independent interface boundary conditions (IBCs).

In this paper a NDD approach is used in which IBCs [1,33-35,37] are applied on the interface between the inner and outer regions. IBCs are calculated using a boundary layer equation in the inner region to transfer the wall boundary condition to the interface. This leads to a Robin type boundary condition at the interface. The full form of the pressure gradient and other source terms can be included in the calculation of the IBCs.

IBCs can be calculated for both HRN and LRN models. In the case of HRN models, the interface should be located sufficiently far from the wall so that the HRN model is applicable in the outer region. This restriction is analogous to the requirement with wall functions that the near-wall cell centre be in the fully turbulent part of the boundary layer. In the case of LRN models, there is no lower restriction on the location of the interface boundary. As the interface boundary approaches the wall, the IBCs tend to the original wall boundary conditions without any modification.

The LRN solution is optimal in that it offers the highest accuracy available with RANS modelling, however the LRN solution takes the most computation time to calculate. On the other hand, the HRN solution is optimal in that the simulation is fastest, however the accuracy is in general the worst. The NDD solution allows a trade-off to be made between accuracy and computation time. As the distance from the interface boundary to the wall increases the computational time reduces since the mesh size decreases, however the accuracy of the solution also decreases. Hence as $y^{*}$ changes, NDD provides all Pareto solutions, whilst the LRN and HRN solutions represent the two extreme (ideal) solutions.

IBCs are expressed in mesh-independent form and contain no free parameters. The only requirement is an approximation of the turbulent viscosity in the inner region. The more accurate this profile is, so the more accurate the NDD solution will be. Many near-wall turbulent viscosity profiles are based on the wall shear stress $[8,2]$ and produce accurate results in one-dimensional regions of flow. However if there are separation points in a flow, then these profiles fail and other profiles must be used. In this work, a turbulent viscosity profile is chosen that depends on the pressure gradient [14]. This is shown to produce accurate results even in regions of flow recirculation.

Previously IBCs have only been applied to the $k-\varepsilon$ model $[33,35,37]$. The $k-\varepsilon$ model is one of the earliest turbulence models and is known to perform poorly in many situations, such as in regions with adverse pressure gradients or streamline curvature, rotating flows and flows with heat transfer [28,31]. In this paper, IBCs are applied to the $k-\omega$ SST [26], Spalart-Allmaras [32] and $\mathrm{BL}-\overline{v^{2}} / k[4]$ models for the first time.

The paper begins in Section 2 by deriving the IBCs used with the NDD approach. Implementation of IBCs with the three turbulence models used is explained in Section 3. Details of the computational code used in this work are given in Section 4. The four test cases studied in this work are discussed in Section 5. These are: a plane channel flow, two different annulus flows and an asymmetric, two-dimensional diffuser. In the case of the two annuli, heat transfer data are reported. A comparison of the computation time for the NDD approach and conventional LRN models with and without wall functions is given for the case of the diffuser. The conclusions are given in Section 6 .

\section{Near-wall domain decomposition and interface boundary conditions}

The governing RANS equation for a function $\Phi$ can usually be written

$\frac{\partial(\rho \Phi)}{\partial t}+\nabla \cdot(\rho \mathbf{U} \Phi)=\nabla \cdot\left(\Gamma_{\Phi} \nabla \Phi\right)+F$

where $\rho$ is the density, $\mathbf{U}$ is the velocity and $F$ is any source terms. For brevity, the diffusion coefficient for the function $\Phi$ is written as

$\Gamma_{\Phi} \equiv \frac{\mu}{\sigma_{\Phi}}+\frac{\mu_{t}}{\sigma_{t, \Phi}}$

where $\mu$ is the dynamic viscosity, $\mu_{t}$ is the turbulent viscosity, $\sigma_{\Phi}$ is the Prandtl number for $\Phi$ and $\sigma_{t, \Phi}$ is the turbulent Prandtl number for $\Phi$. The function $\Phi$ could be a velocity component, transported scalar or function from a turbulence model. It is assumed that $\Phi$ obeys a Dirichlet boundary condition at the wall.

In the near-wall region, $0 \leqslant y \leqslant y^{*}$, Eq. (1) can be written as

$\frac{\partial}{\partial y}\left(\Gamma_{\Phi} \frac{\partial \Phi}{\partial y}\right)=R_{\Phi}(y)$

The full form of the right hand side, $R_{\Phi}(y)$, contains wall-parallel diffusion, the convection terms and any source terms. In many situations, it is a good-enough approximation to ignore wall-parallel diffusion and convection in the inner region $[12,23,30]$. This approximation is made in this work. Under such approximations, the form of $R_{\Phi}$ for the $U$ velocity component is $R_{U}(y)=P_{x}$, where $P_{x}$ is the streamwise pressure gradient. It is stressed, however, that convection and wall-parallel diffusion need not be neglected. In contrast, conventional wall functions, such as the SWF, typically require that $R_{\Phi}=0$. 
The NDD method is formulated by assuming that Eq. (1) applies in the outer domain and that Eq. (3) applies in the inner domain. Boundary conditions are required that link the solutions in the two regions at the interface, which is located at $y^{*}$. These are computed by using Eq. (3) to transfer the boundary conditions for $\Phi$ from the wall to $y^{*}$. The solution can then be computed in the outer region. If it is required, the inner region can be resolved after the solution in the outer region has been computed. The method used to transfer the boundary conditions to the interface is now described.

At each iteration, there is an approximate solution to Eq. (1) in the outer region. Therefore Eq. (3) can be integrated from $y^{*}$ down to a coordinate $y$ in the inner region:

$\Gamma_{\Phi}(y) \frac{\partial \Phi}{\partial y}=\left.\Gamma_{\Phi}^{*} \frac{\partial \Phi}{\partial y}\right|^{*}-\int_{y}^{y^{*}} R_{\Phi} d y^{\prime}$

where a superscript $*$ denotes quantities evaluated at $y^{*}$. Second integration of Eq. (3) is possible using the Dirichlet boundary conditions at the wall. The limits of integration are the wall, where $y=0$, and $y^{*}$ which yields

$\Phi^{*}=\left.\frac{\partial \Phi}{\partial y}\right|^{*} \int_{0}^{y^{*}} \frac{\Gamma_{\Phi}^{*}}{\Gamma_{\Phi}(y)} d y-\int_{0}^{y^{*}} \frac{\int_{y}^{y^{*}} R_{\Phi} d y^{\prime}}{\Gamma_{\Phi}(y)} d y+\Phi_{w}$,

where the subscript $w$ refers to quantities evaluated at the wall. Eq. (5) can be expressed concisely as

$\Phi^{*}=f_{1}^{\Phi} \Phi_{y}^{*}+\tilde{f}_{2}^{\Phi}$

where

$f_{1}^{\Phi}=\int_{0}^{y^{*}} \frac{\Gamma_{\Phi}^{*}}{\Gamma_{\Phi}(y)} d y$

$\tilde{f}_{2}^{\Phi}=-\int_{0}^{y^{*}} \frac{\int_{y}^{y^{*}} R_{\Phi} d y^{\prime}}{\Gamma_{\Phi}(y)} d y+\Phi_{w}$.

Eq. (6) is a Robin boundary condition for $\Phi$ at $y^{*}$ that is used in the outer region.

Engineering flows are often specified in terms of the bulk Reynolds number which requires knowledge of the mass flow rate $\dot{m}$ through a chosen surface. Calculation of $\dot{m}$ requires full knowledge of the velocity profile across the inner regions of the flow. Conventional wall functions treat the velocity in the near-wall cell as a constant, and the mass flow is calculated accordingly. This piecewise-constant form of the velocity profile is incorrect, and the error is largest in the near-wall regions, especially for coarse near-wall cells which are often required with wall function approaches.

When IBCs are used, the full solution $U(y)$ in the inner region can be calculated once the solution in the outer region has been obtained with the expression

$U(y)=\left.\int_{0}^{y} \frac{\Gamma_{U}^{*}}{\Gamma_{U}(\xi)} \frac{\partial U}{\partial y}\right|_{y^{*}} d \xi-\int_{0}^{y} \frac{\int_{y}^{y^{*}} R_{U} d \eta}{\Gamma_{U}(\xi)} d \xi$,

where $\Gamma_{U}=\mu+\mu_{t}$. This equation can be used to calculate $\dot{m}$.

\subsection{IBC for the wall normal velocity}

The wall-normal velocity component, $V$, is of particular importance since the continuity equation must be satisfied over the computational domain. For instance, for the diffuser studied in Section 5.3, the total mass flux across each interface must be zero.

The gradient of $V$ in the wall normal direction $n$ at the wall is known a priori from the continuity equation to be identically zero. The impermeability condition provides a second boundary condition. The right-hand side $R_{V}$ is assumed to be of the form $R_{V}=4 K y^{3}$, for some constant $K$. These conditions allow Eq. (3) to be integrated. The boundary condition for $V$ is

$V\left(y^{*}\right)=K \int_{0}^{y^{*}} \frac{\xi^{4} d \xi}{\mu+\mu_{t}(\xi)}-\frac{\dot{m}_{S}}{\rho S}$

The first term on the right hand side of Eq. (10) comes from integrating Eq. (3). The value of $K$ is chosen to ensure that $V$ is continuous at $y^{*}$ between the inner and outer regions.

The second term on the right hand side of Eq. (10) is a correction term that ensures that the total flux across each interface boundary is zero. The surface area of the interface boundary is $S$ and $\dot{m}_{S}$ is the surface integral of the first term on the right hand side of Eq. (10) over the interface boundary.

\subsection{Near-wall turbulent viscosity profile}

The boundary conditions depend on the diffusion coefficient $\Gamma_{\Phi}$ over the inner region. This depends on $\mu_{t}$, which is not known a priori. A sufficiently accurate profile of $\mu_{t}$ should be used to calculate the boundary conditions.

Many near-wall turbulent viscosity profiles have been proposed in the literature. For example, Craft et al. [12] used a piecewise linear viscosity profile, in which the viscosity is zero in a thin layer near to the wall, to represent the viscous sublayer. The van Driest damping function [38] has been used to build near-wall turbulent viscosity profiles, such as that of Cabot and Moin [8]. In some circumstances an exact profile of the turbulent viscosity is known a priori. For example, in a zero pressure gradient boundary layer the Spalart-Allmaras governing equations can be solved analytically and a turbulent viscosity profile is obtained [2].

When choosing a near-wall turbulent viscosity profile there is a trade-off between accuracy and generality. In this work, the profile of Duprat et al. [14] has been used. The profile is based on the van Driest damping function but is additionally sensitised to the streamwise pressure gradient. The profile was chosen because it was found to produce accurate-enough results in flows with pressure gradients and recirculation regions. Simpler profiles $[2,8]$ are often invalid in such regions and are therefore unsuited for use in a general purpose solver.

Duprat et al. [14] introduced and used this profile in a near wall model as part of a large eddy simulation of flow over periodic hills. Including the pressure gradient in the near-wall turbulent viscosity profile led to a more accurate prediction of the locations of separation and reattachment [14].

The viscosity is calculated as

$\mu_{t}(\xi)=\mu \kappa \xi^{*}\left[\alpha+\xi^{*}(1-\alpha)^{\frac{3}{2}}\right]^{\beta}\left(1-\exp \left(\frac{-\xi^{*}}{1+A \alpha^{3}}\right)\right)^{2}$,

$\kappa=0.41, A=17, \beta=0.78, \alpha=u_{\tau}^{2} / u_{\tau p}^{2} \quad$ and $\quad \xi^{*}=y u_{\tau p} \rho / \mu$. The velocity scales are

$u_{\tau p}=\sqrt{u_{\tau}^{2}+u_{p}^{2}}$,

$u_{\tau}=\sqrt{\left|\tau_{w}\right| / \rho}$,

$u_{p}=\left|\frac{\mu}{\rho^{2}} \frac{\partial P}{\partial x}\right|^{\frac{1}{3}}$

where $\tau_{w}$ is the wall shear stress and $\mu$ is its dynamic viscosity.

The wall shear stress can be obtained from Eq. (4) as

$\tau_{w}=\mu \frac{\partial U}{\partial y}=\frac{\Gamma_{U}^{*}}{f_{1}^{U}}\left(U^{*}-\tilde{f}_{2}\right)-\int_{0}^{y^{*}} R_{U} d y$. 
Its value is updated at each iteration as the solution in the outer region advances.

\subsection{IBCs for passive scalars}

The fluid velocity obeys a Dirichlet boundary condition at the wall. However, passive scalars can obey either Dirichlet or Neumann boundary conditions at the wall. In the case of the temperature, a Dirichlet boundary condition corresponds to a constant wall temperature, and a Neumann boundary condition corresponds to a constant heat flux. The same analysis as in Section 2 can be followed for any scalars that obey Dirichlet boundary conditions.

For Neumann boundary conditions the derivation is simplified. Integration of Eq. (3) is possible because the wall heat flux $\dot{q}_{w}^{\prime \prime}$ is known. The result is

$\Gamma_{\Phi}\left(y^{*}\right) \frac{\partial \Phi}{\partial y}=\dot{q}_{w}^{\prime \prime}+\int_{0}^{y^{*}} R_{\Phi} d \xi$

This is a Neumann boundary condition that is applied at $y^{*}$ in the outer domain.

In many circumstances it is necessary to calculate the bulk temperature, $T_{b}$ in order to calculate, for example, the Nusselt number. This requires a knowledge of the profile of $T$ across the inner regions, which can be calculated using the solution in the outer region and Eq. (16) as

$\Phi(y)=\Phi^{*}-\int_{y}^{y^{*}} \frac{\dot{q}_{w}^{\prime \prime}}{\Gamma_{\Phi}(\xi)} d \xi-\int_{y}^{y^{*}} \frac{\int_{0}^{y^{*}} R_{\Phi} d \eta}{\Gamma_{\Phi}(\xi)} d \xi$.

This profile is updated at every iteration in the outer region. Integration of this profile allows for a more accurate calculation of $T_{b}$ than is possible with conventional wall functions, which assume that the temperature is constant in the near-wall cell.

\section{Application of the NDD approach to LRN turbulence models}

Previously, IBCs have only been applied to the $k-\varepsilon$ turbulence model $[33-35,37]$. For the $k-\varepsilon$ the dissipation $\varepsilon$ can be modelled in the inner region as [37]

$\varepsilon(y)=\max \left(\frac{\left(k^{*}\right)^{3 / 2}}{c_{l} y}, \frac{2 \mu k^{*}}{\rho y^{2}}\right)$,

where $c_{l}=2.55$.

An advantage of using IBCs is that $k$ can be given a Robin boundary condition at the interface. This is achieved by writing the $k$ equation in the inner region as

$\frac{\partial}{\partial y}\left(\Gamma_{k} \frac{\partial k}{\partial y}\right)=-\rho \mathcal{P}+\rho \varepsilon$.

The production $\mathcal{P}$ is approximated as

$\rho \mathcal{P} \equiv-\rho \overline{u_{i} u_{j}} \frac{\partial U_{i}}{\partial x_{j}} \approx-\rho \overline{u v} \frac{\partial U}{\partial y} \approx \mu_{t}\left(\frac{\partial U}{\partial y}\right)^{2}$

The required velocity gradient is calculated using Eq. (4). This means that the right-hand side of the $k$ equation is known across the inner region which allows a Robin boundary condition to be calculated at $y^{*}$ :

$k^{*}=f_{1}^{k} k_{y}+\tilde{f}_{2}^{k}$.

In this work the domain decomposition approach is applied to three LRN turbulence models: the Spalart-Allmaras, $k-\omega$ SST and $\mathrm{BL}-\overline{v^{2}} / k$ turbulence models. The full governing equations of the three models are given in Appendix A.
The $k-\omega$ SST and Spalart-Allmaras models require the distance of each cell centre from the wall as a parameter. When using the NDD approach it is necessary to modify the calculation of this parameter to account for the offset between the interface boundary and the physical wall.

\subsection{IBCs for the Spalart-Allmaras model}

The boundary conditions for $\tilde{v}$ come directly from the turbulent viscosity profile used in the inner region. The turbulent viscosity is calculated with the Spalart-Allmaras model as $\mu_{t}=\rho \tilde{v} f_{v 1}$, which can be expressed explicitly in terms of $\tilde{v}$ and $v \equiv \mu / \rho$ as

$\mu_{t}=\frac{\rho \tilde{v}\left(\frac{\tilde{v}}{v}\right)^{3}}{\left(\frac{\tilde{v}}{v}\right)^{3}+c_{v 1}^{3}}$.

At each iteration, the boundary conditions on $\tilde{v}$ are calculated using the fixed point iterative procedure

$\tilde{v}_{i+1}=F\left(\tilde{v}_{i}\right)=\frac{\mu}{\rho}\left(\frac{\mu_{t}\left(\frac{\rho^{3} \tilde{v}_{i}^{3}}{\mu^{3}}+c_{v 1}^{3}\right)}{\rho \tilde{v}_{i}}\right)^{1 / 3}$.

The iterative procedure is initialised with the guess $\tilde{v}_{0}=\mu_{t} / \rho$ and typically converges to good enough accuracy in fewer than 10 iterations.

\subsection{IBCs for the $k-\omega$ SST model}

The boundary conditions on $k$ are calculated by assuming that the turbulent kinetic energy in the $k-\omega$ SST model corresponds to that in the $k-\varepsilon$ model. Therefore Eq. (19) is taken to hold in the inner region and Eq. (21) is applied to $k$.

The $\omega$ boundary condition is specified using the blending approach of Knopp et al. [23]. The solutions of the $\omega$ equation in the viscous sub layer, $\omega_{v}$, and in the logarithmic region of the turbulent boundary layer, $\omega_{l}$, are blended to determine $\omega$ across the inner region. The required definitions are

$\omega_{v}=\frac{6 \mu}{\rho \beta_{1} y^{* 2}}$,

$\omega_{l}=\frac{u_{\tau}}{\sqrt{\beta_{2}} \kappa y^{*}}$

$\phi=\tanh \left(\zeta^{4}\right)$,

$\zeta=y^{*+} / 10$

$\omega_{b 1}=\omega_{v}+\omega_{l}$

$\omega_{b 2}=\left(\omega_{v}^{1.2}+\omega_{l}^{1.2}\right)^{1 / 1.2}$,

which allow $\omega$ to be given the Dirichlet boundary condition

$\omega^{*}=\phi \omega_{b 1}^{*}+(1-\phi) \omega_{b 2}^{*}$,

at $y^{*}$.

\subsection{IBCs for the $\mathrm{BL}-\overline{v^{2}} / k$ model}

The dissipation in the BL- $\overline{v^{2}} / k$ model, $\varepsilon_{h}$, is different to the dissipation $\varepsilon$ in the $k-\varepsilon$ model. Therefore the boundary condition given by Eq. (18) cannot be used.

The $\varepsilon_{h}, \varphi$ and $\alpha$ equations each contain non-linear terms which are important in the near-wall region and cannot be neglected. Therefore, without further knowledge of the form of these 
non-linear terms, IBCs cannot be derived for these functions. Instead, the adaptive wall function of Billard et al. [5] is modified for use in a domain decomposition approach.

The wall function of Billard et al. [5] is based on the look-up table approach of Kalitzin et al. [20]. The turbulence governing equations are made non-dimensional by writing

$\varepsilon_{h}^{+}=\frac{\mu \varepsilon_{h}}{\rho u_{k}^{4}}$,

and using the non-dimensional distance $y^{+}$. The functions $\varphi$ and $\alpha$ are already non-dimensional. In non-dimensional form the turbulence functions are less sensitive to changes in Reynolds number [6].

The non-dimensional distance $y^{+}$is calculated with a cubic spline interpolation:

$$
\begin{aligned}
y^{+}= & \left(2 t^{3}-3 t^{2}+1\right) f_{i}+\left(t^{3}-2 t^{2}+t\right) m_{i} \Delta x_{i}+\left(3 t^{2}-2 t^{3}\right) f_{i+1} \\
& +\left(t^{3}-t^{2}\right) m_{i+1} \Delta x_{i}
\end{aligned}
$$

where the argument $x=k y^{2} / v^{2}, t=\left(x-x_{i}\right) / \Delta x, \Delta x=x_{i+1}-x_{i}$ and $x_{i}<x<x_{i+1}$. The interpolation data $x_{i}$ and $f_{i}$ are given in Table 1, which is taken from Billard et al. [5].

Analytical solutions are sought for $\varepsilon_{h}^{+}$and $\varphi$ in the viscous sub layer and logarithmic region of the boundary layer. The analytical solutions contain constants that are tuned to the solution of a plane channel flow at $R e_{\tau}=1020$, obtained with a LRN computation. The channel flow solution is also used to build cubic spline interpolations for $\varepsilon_{h}^{+}$and $\varphi$ for the buffer layer between the viscous sub layer and the logarithmic region.

For $\alpha$, an analytical solution is found near to the wall by assuming that the turbulent length scale is constant for $y^{+}<17$. The channel flow solution is used to build an empirical expression for $\alpha$ above this limit.

The solutions for $\varepsilon_{h}, \varphi$ and $\alpha$ are [5]:

$\varepsilon_{h}^{+}= \begin{cases}\frac{500 c_{T}^{2}}{c_{\varepsilon_{h}}\left(y^{+}+12.43\right)^{4}} & \text { if } y^{+}<1, \\ \mathfrak{\mho}_{\varepsilon_{h}}\left(y^{+}\right) & \text {if } 1 \leqslant y^{+}<30, \\ \frac{1}{\kappa y^{+}} & \text {if } 30 \leqslant y^{+},\end{cases}$

$\varphi= \begin{cases}0.0015 y^{+2} & \text { if } y^{+}<1, \\ \mathfrak{F}_{\varphi}\left(y^{+}\right) & \text {if } 1 \leqslant y^{+}<30, \\ 0.1336 \log \left(y^{+}\right)-0.2775 & \text { if } 30 \leqslant y^{+},\end{cases}$

$\alpha= \begin{cases}1-\exp \left(\frac{-y^{+}}{24.52}\right) & \text { if } y^{+} \leqslant 17, \\ \left(1+\left(\frac{17}{y^{+}}\right)^{4 / 3}\right)^{-1} & \text { if } 17<y^{+} .\end{cases}$

The functions $\mathfrak{F}_{\varepsilon_{h}}$ and $\mathfrak{F}_{\varphi}$ are spline interpolation functions. The interpolation formula, written in terms of $s=\left\{\varepsilon_{h}^{+}, \varphi\right\}$, is [5]

$$
\begin{aligned}
\mathfrak{F}_{s}\left(y^{+}\right)= & \left(2 t^{3}-3 t^{2}+1\right) f_{i}^{s}+\left(t^{3}-2 t^{2}+t\right) m_{i}^{s} \Delta y_{i}^{+}+\left(3 t^{2}\right. \\
& \left.-2 t^{3}\right) f_{i+1}^{s}+\left(t^{3}-t^{2}\right) m_{i+1}^{s} \Delta y_{i}^{+},
\end{aligned}
$$

where

$t=\frac{1}{\Delta y^{+}} \log \left(\frac{y^{+}}{y_{i}^{+}}\right)$

$\Delta y^{+}=\log \left(\frac{y_{i+1}^{+}}{y_{i}^{+}}\right)$,

and $y_{i}^{+}<y^{+}<y_{i+1}^{+}$. Eq. (36) uses the data in Table 2, which is taken from Billard et al. [5].

It is important to use the correct definition of $y^{+}$for the $\varepsilon_{h}, \varphi$ and $\alpha$ boundary conditions, otherwise the spline interpolations will be incorrect.

Eqs. (33)-(35) are used to impose Dirichlet boundary conditions at the interface at $y^{*}$.

The $k$ boundary conditions for the $\mathrm{BL}-\overline{v^{2}} / k$ model are calculated with the same procedure as they are for the $k-\omega$ SST model. However in this case Eq. (33) is used for $\varepsilon_{h}$ and the additional "E term" in the $k$ equation (Eq. (A.32)) is computed using the wall function of Billard et al. [5]. Firstly a non-dimensional quantity $E^{+}$is calculated as

$E^{+}(y)=0.0875 \exp \left[-\left(\log _{10}\left(y^{+}\right)-1.01\right)^{2} / 0.0484\right]$,

which is converted into a dimensional form as $E(y)=E u_{k}^{4} \rho / \mu$ [5].

\section{Computational code}

The computational code used in this work is Code_Saturne [3], which is an open-source finite volume-based solver developed by EDF R\&D. Convection terms are discretised using second-order central differencing. Gradients are calculated using an iterative method with a reconstruction method for non-orthogonalities in the mesh. The pressure correction procedure uses a modified SIMPLEC algorithm. The pressure equation is solved using the conjugate gradient method. All other variables are solved for with the Jacobi method. In the case of periodic flows, the flow is driven by a momentum source term which is adjusted at each iteration in order to maintain the desired mass flow rate. When the temperature equation is solved, the Prandtl number is taken as $\sigma=0.71$ and the turbulent Prandtl number is $\sigma_{t}=1$. Thermal turbulent heat fluxes are calculated using a gradient diffusion hypothesis.

In all simulations, an unsteady algorithm is used with the pseudo time step chosen to give a maximum cell Courant number of 1 . Since the NDD meshes are created from the LRN meshes with the near-wall cells removed, the smallest cell volume in the NDD mesh is always larger than it is in the LRN mesh. Therefore it is possible to use a larger pseudo-time step in the simulations, whilst retaining the same maximum Courant number.

The scalable wall function is used with the $k-\omega$ SST model. The implementation of this method in Code_Saturne is outlined here. The wall shear stress is written $\tau_{w}=\rho u_{k} u_{\tau}$, where $u_{k}=c_{\mu}^{1 / 4} k^{1 / 2}$, with $c_{\mu}=0.09$, and

$u_{\tau}=\frac{U_{I}}{\frac{1}{\kappa} \ln \left(y^{+}\right)+C}$,

where $U_{I}$ is the wall parallel velocity at the near-wall cell centre,

\begin{tabular}{|c|c|c|c|c|c|c|c|c|}
\hline$i$ & 1 & 2 & 3 & 4 & 5 & 6 & 7 & 8 \\
\hline$x_{i}$ & 0 & 0.5 & 5 & 40 & 300 & 1000 & 10,000 & 100,000 \\
\hline$f_{i}$ & 0 & 1.53 & 2.846 & 5.058 & 9.926 & 16.21 & 53.64 & 217.451 \\
\hline$m_{i}$ & 10 & 0.77 & 0.143 & 0.0386 & 0.0123 & 0.007295 & 0.003025 & 0.00159 \\
\hline
\end{tabular}
$y^{+}=y \rho u_{k} / \mu$, with $y$ the distance of the near-wall cell centre from

Table 1

The data used to interpolate $y^{+}$for the BL- $\overline{v^{2}} / k$ model [5]. 
Table 2

Data used to interpolate $\varepsilon_{h}^{+}$and $\varphi$ between $1 \leqslant y^{+}<30$ [5].

\begin{tabular}{llllll}
$i$ & 1 & 2 & 3 & 4 & 5 \\
$y_{i}^{+}$ & 1 & 3 & 5 & 11 & 30 \\
$f_{i}^{\varepsilon_{h}^{+}}$ & 0.0732 & 0.063 & 0.091 & 0.149 & 0.0793 \\
$m_{i}^{\varepsilon_{h}^{+}}$ & -0.021 & 0.025 & 0.082 & 0 & -0.074 \\
$f_{i}^{\varphi}$ & 0.00147 & 0.008 & 0.0165 & 0.0596 & 0.177 \\
$m_{i}^{\varphi}$ & 0.002299 & 0.0123 & 0.0264 & 0.088 & 0.138 \\
\hline
\end{tabular}

the wall, and $C=5.2$. A further boundary condition is applied to the velocity to ensure that the gradient at the centre of the near-wall cell is greater than or equal to $u_{\tau} /(\kappa y)$. The turbulent kinetic energy receives a homogeneous Neumann boundary condition, and $\omega$ is prescribed the following Neumann boundary condition:

$\frac{d \omega}{d y}= \begin{cases}\frac{4 y u_{k}^{3} \rho^{2}}{c_{\mu}^{1 / 2} \kappa \mu^{2}\left(y^{+}\right)^{2}}, & \text { if } y^{+}>2.38, \\ \frac{960 \mu}{\rho \beta_{1} y^{2}}, & \text { otherwise. }\end{cases}$

The full set of equations for all turbulence models used in this work can be found in Appendix A along with the boundary conditions used at the wall in LRN simulations.

\section{Test cases}

The NDD method is tested on a plane channel flow, two heated annulus flows at different Reynolds numbers and an asymmetric, two-dimensional diffuser flow.

In every case a LRN mesh is first created and mesh independence is obtained. The NDD meshes are then created by removing any cells from the LRN mesh that lie within the inner regions of the NDD domain. What remains in the mesh defines the outer region and the cell distribution is identical to the LRN mesh in this part of the domain. The NDD solutions were tested separately for mesh independence, and were found to be independent in every case using this procedure. Meshes for the HRN simulations were created such that the near-wall cell centre was located at the same $y^{*}$ as the interface boundary condition.

\subsection{Plane channel flow test case}

The first test case of the NDD approach is a statistically one-dimensional plane channel flow with Reynolds number based on the friction velocity $u_{\tau}$ and channel half height $h$ of $R e_{\tau}=590$. Results are compared to the DNS velocity profile of Moser et al. [27].

The solution is obtained on a one-dimensional mesh with a wall located at $y=0$ and a symmetry boundary condition imposed at $y=h$. Periodic boundary conditions are applied in the stream-wise $x$ direction, and a symmetry boundary condition is applied in the $z$ direction. The flow is driven by a constant momentum source term, $\mathcal{F}$, which for this particular case is related to the wall shear stress as

$\tau_{w}=h \mathcal{F}$.

Different simulations are performed with the NDD approach with interfaces located at $y^{*} / h=0.01,0.03,0.05$ and 0.1 , which gives a $y^{+}$range of 5.9 to 59 .

The profiles of $U^{+} \equiv U / u_{\tau}$ obtained with each turbulence model are shown in Fig. 1. The NDD solution is seen to be in good agreement with the DNS and LRN solutions in every case. The velocity profile in the viscous sub layer for $y^{+}<5$ shows no sensitivity to $y^{*}$, even for the cases with $y^{*+}>5$ where the solution in the viscous sub layer is calculated with Eq. (9). This demonstrates that

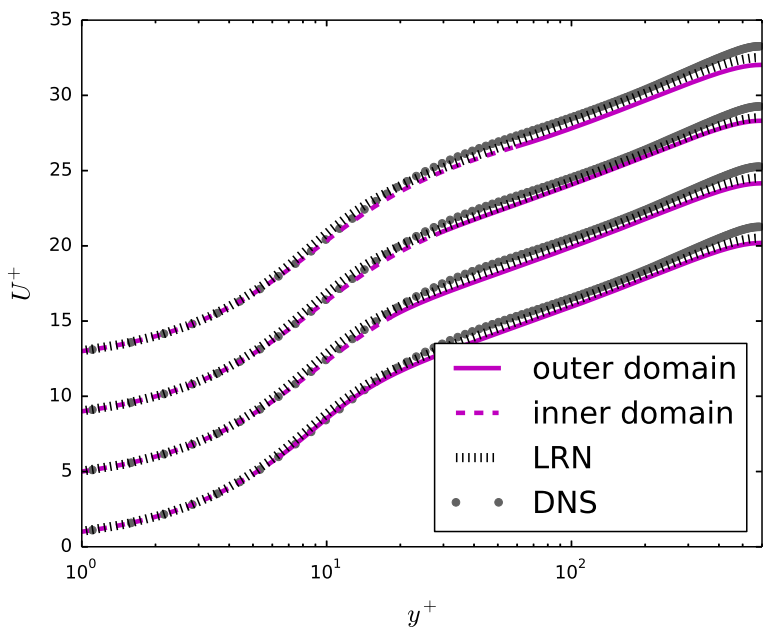

(a) Spalart-Allmaras model

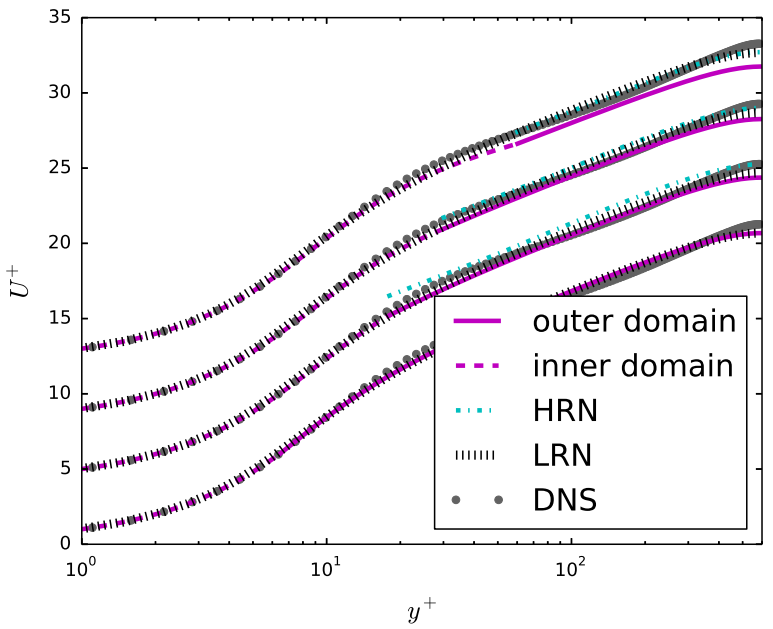

(b) $k-\omega$ SST model

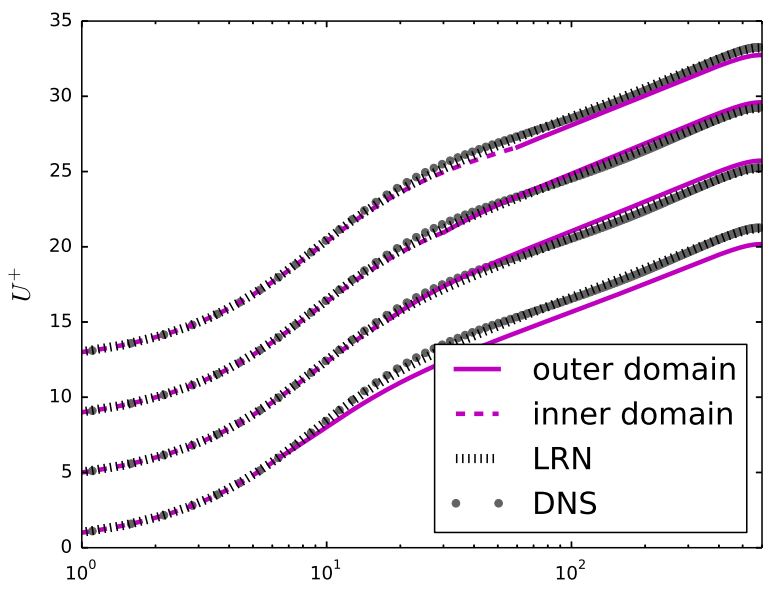

(c) $\mathrm{BL}-\frac{y^{+}}{v^{2}} / k$ model

Fig. 1. $U^{+}$in the channel flow obtained with each turbulence model. The DNS data is that of Moser et al. [27]. For the NDD results, the inner region is represented by the dashed line; the solid line represents the outer region. The results at different $y^{*}$ are shifted along the vertical axis for clarity. The lowest curves are the data for $y^{*} / h=0.01$ and the highest curves are the data for $y^{*} / h=0.1$.

near-wall damping effects are correctly captured with the NDD approach. 
In Fig. $1 \mathrm{~b}$ the HRN $k-\omega$ SST results are shown. The NDD results appear to be slightly more accurate near to the wall, and the HRN results slightly more accurate near the channel centreline. However the variation with $y^{*}$ for the two approaches is small.

\subsection{Annulus flows}

Annulus flows occur in engineering applications such as pipeline flows or heat exchangers. Because of this, many experiments were performed in the 1950s and 1960s, with the aim of developing a method to predict the Nusselt number at each wall. For example, Davis [13] developed a numerical correlation for a range of Reynolds numbers, radius ratios and Prandtl numbers, whereas Kays and Leung [22] produced tabulated data for a range of Reynolds numbers and radius ratios. With the increase in computational power in recent years it is now possible to perform DNS studies of annulus flows, although only at Reynolds numbers well below those that commonly occur in engineering applications. Examples include the study of Chung et al. [9], who studied the effect of the radius ratio and the study of Ould-Rouiss et al. [29], who studied the effect of the ratio of heat fluxes at the two walls. Annulus correlations remain a topic of current research. For example, Yu et al. [40] studied flow in an annulus with a heated inner wall and an adiabatic outer wall. A numerical correlation was built by integrating the energy equation, using a model for the turbulent stresses. In this way a correlation for the Nusselt number that is more accurate than early experimental studies was obtained.

Annulus flows are characterised by the ratio of the outer and inner radii, $\alpha \equiv R_{2} / R_{1}$, and the heat flux ratio between the outer and inner walls, $q^{*} \equiv \dot{q}_{2}^{\prime \prime} / \dot{q}_{1}^{\prime \prime}$, where the subscript 1 refers to the

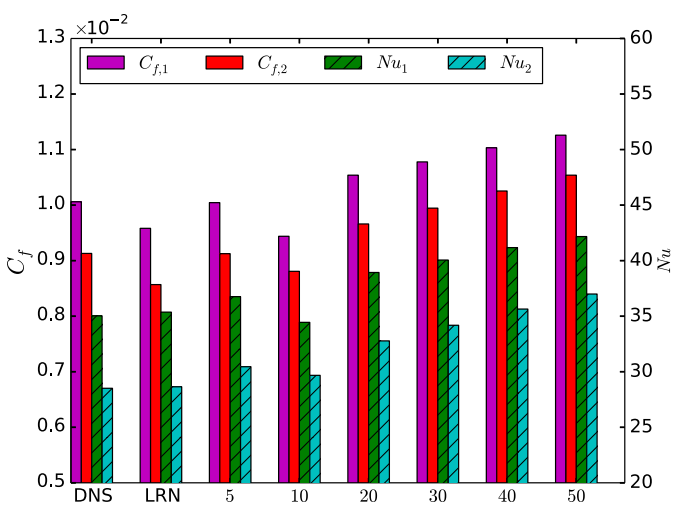

(a) Spalart-Allmaras with NDD

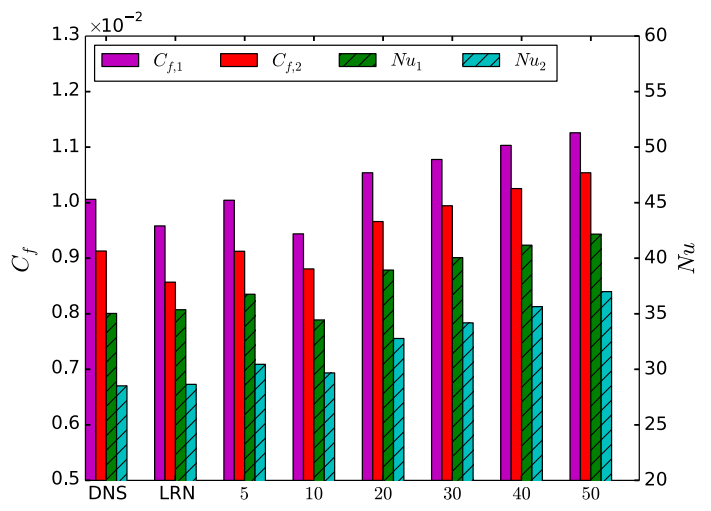

(b) $k-\omega \mathrm{SST}$ with NDD

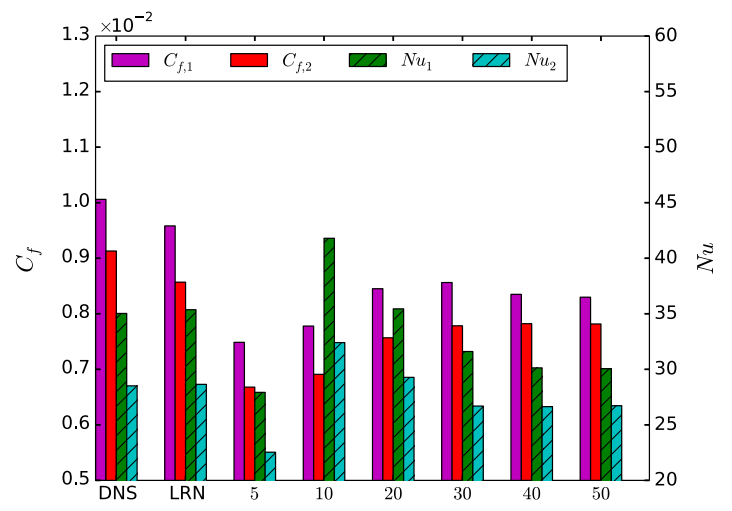

(c) HRN $k-\omega \mathrm{SST}$

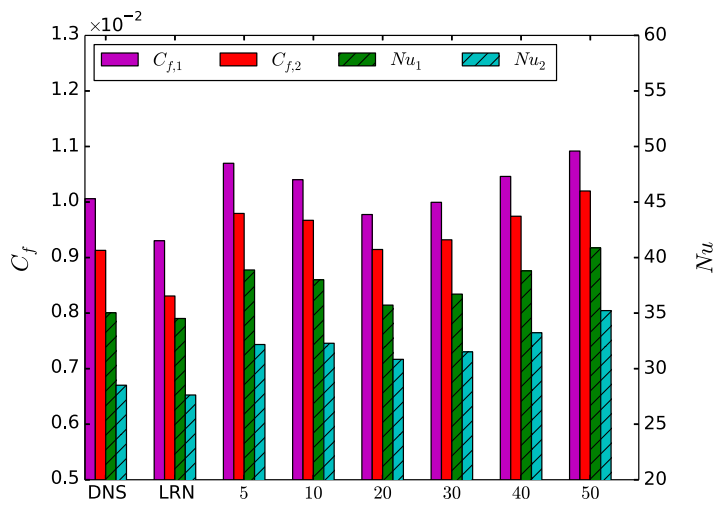

(d) $\mathrm{BL}-\overline{v^{2}} / k$ with NDD

Fig. 2. $C_{f}$ and $\mathrm{Nu}$ on both walls for the annulus flow at $R e=8.9 \times 10^{3}$. The number on the $x$ axis are equal to $10^{3} y^{*} / d_{e}$ for the NDD and HRN cases. 
inner wall and 2 to the outer wall. The Reynolds number is specified in terms of the equivalent diameter $d_{e}=2\left(R_{2}-R_{1}\right)$.

The first annulus flow has $\alpha=2, q^{*}=1$ and $R e=8.9 \times 10^{3}$. A direct numerical simulation (DNS) of this case has been performed by Chung and Sung [10]. The second annulus flow has $\alpha=52.07 / 26.67, q^{*}=0$ and a range of Reynolds numbers from $R e=2.58 \times 10^{5}$ to $1.125 \times 10^{6}$. These conditions are similar to those which might occur in an engineering application. Comparison is made to the experimental data of Watson [39], who studied heat transfer in an annulus as part of a project to determine the optimum geometry of a nuclear reactor fuel assembly. In each case the flow is studied with the NDD method for three different locations of the interface boundary.

All of the annulus flows are set up such that the flow velocity is $\mathbf{U}=(0,0, W(r))$. Periodic boundary conditions are applied in the $z$ direction. The meshes always contain a $1^{\circ}$ slice of the annulus. Rotational periodicity is used to determine the boundary conditions in the azimuthal direction.

The flows are driven by a constant momentum source term $\mathcal{F}$, which acts as the pressure gradient with $\mathcal{F}=-P_{z}$. This source term is determined at every iteration by computing the bulk Reynolds number, and adjusting the value of $\mathcal{F}$ until convergence is achieved at the desired Reynolds number.

When NDD is used, in every case the inner and outer wall interfaces are the same distance from their respective walls.

\subsubsection{Annulus flow at $\operatorname{Re}=8.9 \times 10^{3}$}

The first of the two annulus flows is that of Chung et al. [9] and Chung and Sung [10], who performed a DNS of the flow between two heated pipes. The Reynolds number based on the equivalent diameter $d_{e}$ and bulk velocity $U_{b}$ is $R e=8.9 \times 10^{3}$. The radius ratio is $\alpha=2$. Both pipes are heated with the same constant heat flux such that $q^{*}=1$.

NDD simulations are performed with interfaces located at $y^{*} / d_{e}=0.005,0.01,0.02,0.03,0.04$, and 0.05 .

The skin friction coefficient is $C_{f}=2 \tau_{w} /\left(\rho U_{b}^{2}\right)$ and the Nusselt number is

$N u=\frac{q_{w}^{\prime \prime} d_{e} \sigma}{\mu c_{p}\left(T_{w}-T_{b}\right)}$,

where $T_{w}$ is temperature at the wall and $c_{p}$ is the specific heat capacity. Fig. 2 shows $C_{f}$ and $N u$ at both walls for all simulations. The HRN $k-\omega$ SST results are shown in Fig. 2c.

The skin friction coefficients obtained with the NDD method are in agreement with the LRN and DNS values. The skin friction coefficients tend to be slightly larger than the LRN results when

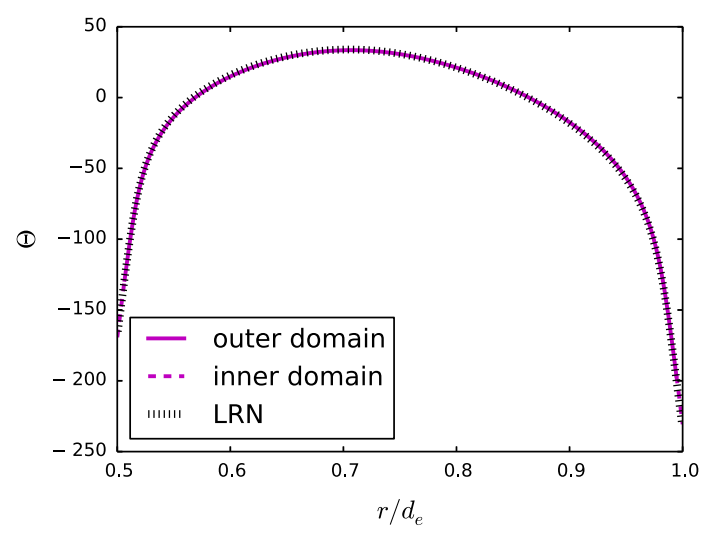

Fig. 3. $\Theta$ in the annulus flow at $R e=8.9 \times 10^{3}$ with the Spalart-Allmaras model for $y^{*} / d_{e}=0.005$. the NDD approach is used. This makes the NDD skin friction coefficients agree more closely with the DNS results than the LRN calculation. The maximum error in the LRN skin friction coefficients, as a percentage of the DNS value is approximately $17 \%$ for $C_{f 2}$ with the Spalart-Allmaras model at $y^{*} / d_{e}=0.05$. This compares to a maximum error with the HRN $k-\omega$ SST model of approximately $27 \%$ for $C_{f 2}$, with $y^{*} / d_{e}=0.05$. In contrast, the maximum error in the LRN skin friction coefficients is approximately $6 \%$ and occurs with the Spalart-Allmaras model for $C_{f 2}$ at $y^{*} / d_{e}=0.01$.

The Nusselt numbers at both walls are also in good agreement with the LRN and DNS values. The trends in $\mathrm{Nu}$ with $\mathrm{y}^{*}$ are similar to those in $C_{f}$. The maximum error in the NDD Nusselt numbers is approximately $30 \%$ and occurs with the Spalart-Allmaras model at $y^{*} / d_{e}=0.05$. The maximum error with the HRN $k-\omega$ SST model is approximately $20 \%$ and occurs for $y^{*} / d_{e}=0.1$. In contrast the maximum error in the LRN Nusselt numbers is approximately 7\% and occurs with the Spalart-Allmaras model.

As $y^{*}$ becomes larger the skin friction factors and Nusselt numbers tend to increase. However at $y^{*} / d_{e}=0.05$, only $80 \%$ of the total flow domain is covered by the computational mesh. This $y^{*}$ is relatively large and it is unsurprising that the error is largest for this case.

The dimensionless temperature

$\Theta=\frac{\rho c_{p} U_{b}\left(T-T_{b}\right)}{\dot{q}_{w}^{\prime \prime}}$,

calculated with the Spalart-Allmaras model is plotted against the radial coordinate in Fig. 3 for $y^{*} / d_{e}=0.005$. The NDD profile is indistinguishable from the LRN profile. Profiles of $\Theta$ found with the BL- $\overline{v^{2}} / k$ and $k-\omega$ SST models show similar behaviour.

\subsubsection{Annulus flow at larger Reynolds numbers}

The second annulus is that of Watson [39], who performed an experimental study of the flow of carbon dioxide over a heated rod. The radius ratio is $\alpha=52.07 / 26.67 \approx 1.95$. The range of Reynolds numbers based on equivalent diameter and bulk velocity are $R e=2.58 \times 10^{5}, 3.50 \times 10^{5}, 5.08 \times 10^{5}, 7.73 \times 10^{5}, 9.53 \times 10^{5}$, $1.125 \times 10^{6}$. The inner pipe is heated with a constant heat flux; the outer pipe is insulated and the heat flux there is zero. Experimental results are reported as the friction factor

$f=\frac{d_{e}\left|P_{z}\right|}{2 \rho U_{b}^{2}}$,

and Stanton number on the inner wall

$S t=\frac{\dot{q}_{w}^{\prime \prime}}{\rho U_{b} c_{p}\left(T_{w}-T_{b}\right)}$.

NDD simulations are performed with $y^{*} / d_{e} \approx 0.001,0.005$, 0.01 , and 0.02 , which gives a maximum $y^{+} \approx 950$ at $R e=1.125 \times$ $10^{5}$ and a minimum $y^{+} \approx 12$ at $\operatorname{Re}=2.58 \times 10^{5}$. This range of $y^{+}$ is larger than is possible for the first annulus flow. The resulting friction factors are shown in Fig. 4 and the Stanton numbers are shown in Fig. 5.

The NDD friction factors typically vary no more than $10 \%$ from the LRN result as $y^{*}$ varies. The smallest variation with $y^{*}$ is seen in the Spalart-Allmaras results and the largest variation is seen for the $\mathrm{BL}-\overline{v^{2}} / k$ model. The variation in the NDD results with $y^{*}$ is typically similar to the discrepancy between the experimental and LRN results. The trend in $f$ with $R e$ is predicted correctly with every turbulence model with every $y^{*}$. The results for $y^{*} / d_{e}=0.001$ show the least accurate prediction of the trend. This is because at lower Reynolds numbers, this $y^{*}$ is in the buffer region of the boundary layer, whereas it is in the fully turbulent region at higher 
Reynolds numbers. The HRN $k-\omega$ SST friction factors show little variation with $y^{*}$. At lower Reynolds numbers the error is around $5 \%$ of the LRN result, and decreases as the Reynolds number increases.

Similar trends are observed for the Stanton number in Fig. 5. Again, the Spalart-Allmaras model shows the least variation in Stanton number with $y^{*}$ and the BL $-\overline{v^{2}} / k$ shows the largest. The NDD Stanton numbers typically vary by approximately $10 \%$ with the range of $y^{*}$ considered here. In contrast, the HRN $k-\omega$ SST results show little variation with $y^{*}$, however the Stanton numbers are always less than the LRN value, and significantly below the experimental value. This means that the NDD results agree more

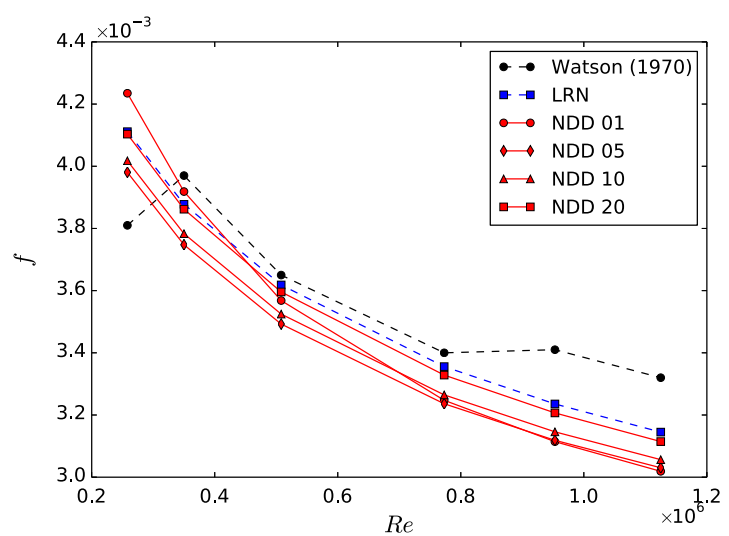

(a) Spalart-Allmaras

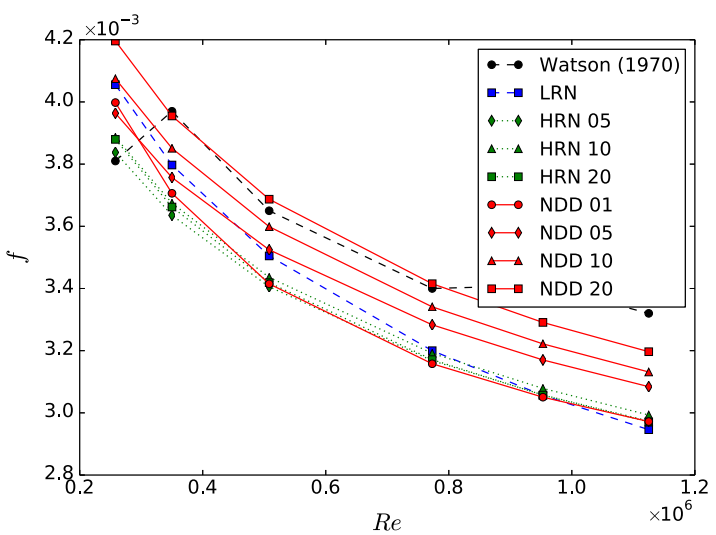

(b) $k-\omega \mathrm{SST}$

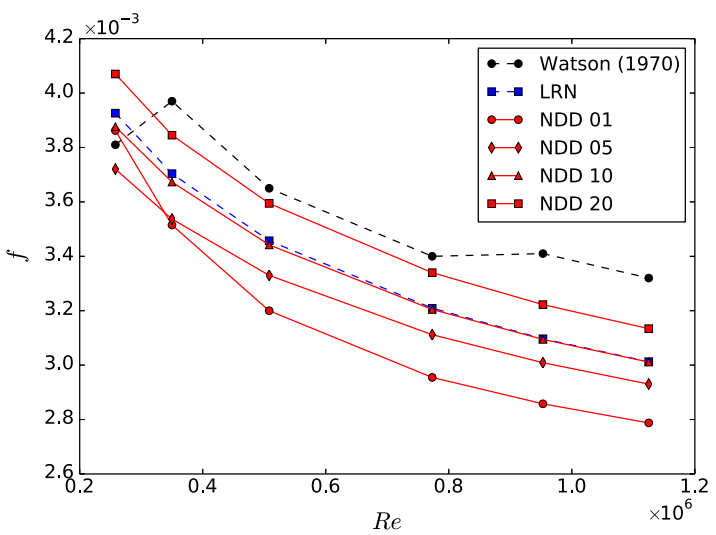

(c) $\mathrm{BL}-\overline{v^{2}} / k$

Fig. 4. $f$ for the annulus flows of Watson [39]. The numbers in the legend are the values of $10^{3} y^{*} / d_{e}$ for the NDD and HRN results. closely with the experimental data than the HRN results do in every case.

The dimensionless temperature

$\zeta=\frac{T-T_{b}}{T_{w}-T_{b}}$

obtained with the Spalart-Allmaras model is plotted in Fig. 6 for the case with $y^{*} / d_{e} \approx 0.001$ and $R e=2.58 \times 10^{5}$. The difference between the LRN and NDD predictions is not visible in these plots. Similar results are found with the profiles of $\zeta$ obtained with the $\mathrm{BL}-\overline{v^{2}} / k$ and $k-\omega$ SST models and at different $y^{*}$ and $R e$.

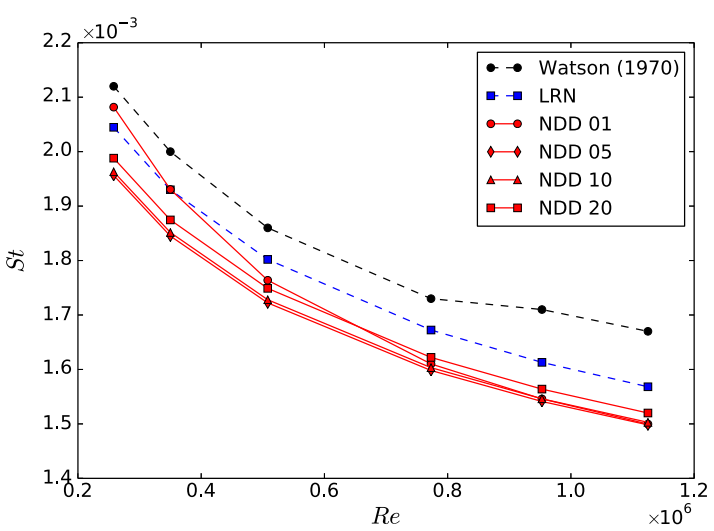

(a) Spalart-Allmaras

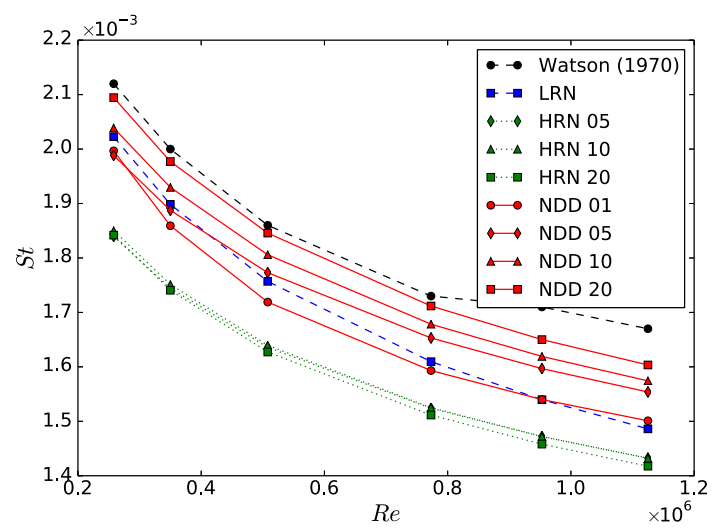

(b) $k-\omega \mathrm{SST}$

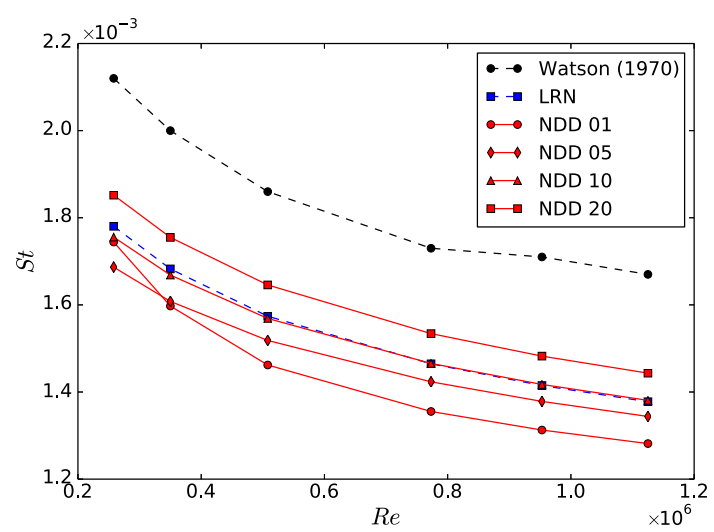

(c) $\mathrm{BL}-\overline{v^{2}} / k$

Fig. 5. St for the annulus flows of Watson [39]. The numbers in the legend are the values of $10^{3} y^{*} / d_{e}$ for the NDD and HRN results. 


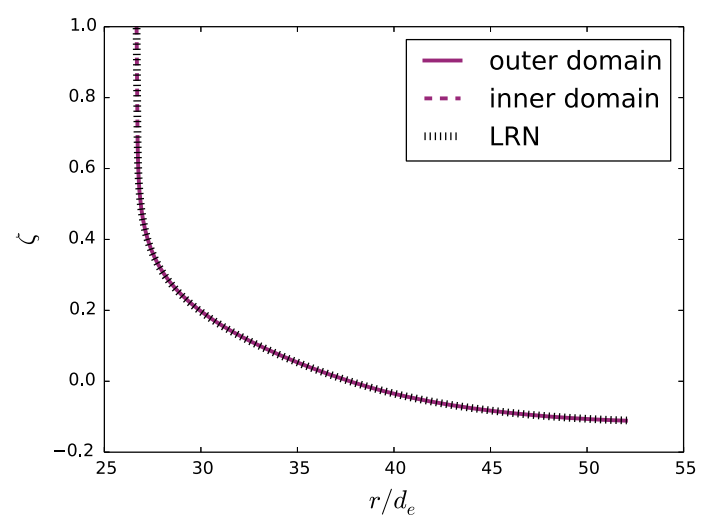

Fig. 6. $\zeta$ in the annulus flow at $R e=2.58 \times 10^{5}$ with the Spalart-Allmaras model for $y^{*} / d_{e} \approx 0.001$

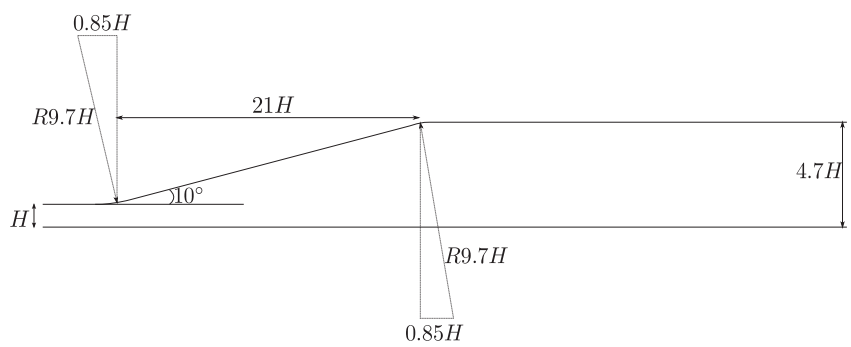

Fig. 7. The geometry of the diffuser.

\subsection{Two-dimensional asymmetric diffuser}

The final test case is that of a two-dimensional asymmetric diffuser, which was studied as part of the 8th ERCOFTAC IAHR workshop [19]. In the workshop a number of RANS turbulence models were tested on the geometry and compared to the experimental data of Buice and Eaton [7]. The geometry has also been studied with LES $[21,19]$. A separation region forms along the inclined wall of the diffuser region which is located in between $x / H=0$ and $x / H=21$.

The diffuser geometry is shown in Fig. 7. The inlet is located at $x / H=-10$, and the flow there is that of a fully developed plane channel flow at $R e=18,000$. The inlet conditions are calculated in a separate LRN calculation. A pressure outlet is located at $x / H=75$. Symmetry boundary conditions are applied in the $z$ direction.

Simulations are performed with the NDD method with cut heights equal to $y^{*} / H=0.01,0.03$ and 0.05 . The cut height is the same on each wall such that for the case with $y^{/} H=0.0590 \%$ of the channel height is meshed. The HRN meshes have the same cell distribution in the $x$ direction however the spanwise mesh resolution is reduced.

The separation and reattachment points are predicted by the turbulence model. They can be visualised via the skin friction coefficient which is shown in Fig. 8 for the three turbulence models along the inclined wall. It is seen that for every turbulence model the NDD result approaches the LRN result as $y^{*}$ is reduced. The NDD results are in agreement with the LRN result for every $y^{*}$ and a recirculation region is predicted in every case. In contrast, the HRN results with the $k-\omega$ SST model do not capture the recirculation region for $y^{*} / H=0.03$ and 0.05 . This is because the $\log$ law does not take into account the pressure gradient and therefore the calculated wall shear stress predicted always has the same sign as the wall-parallel velocity at the near-wall cell centre.

\subsubsection{Comparison of computation time}

Convergence is assessed through a convergence parameter, $D$, which can be calculated for every non-zero variable $\Phi$ at every iteration $k$ with the expression

$D(\Phi)=\frac{1}{\Delta t} \sqrt{\frac{\sum_{i=1}^{N}\left(\Phi_{i}^{k}-\Phi_{i}^{k-1}\right)^{2} \Omega_{i}}{\sum_{i=1}^{N}\left(\Phi_{i}^{k}\right)^{2} \Omega_{i}}}$.

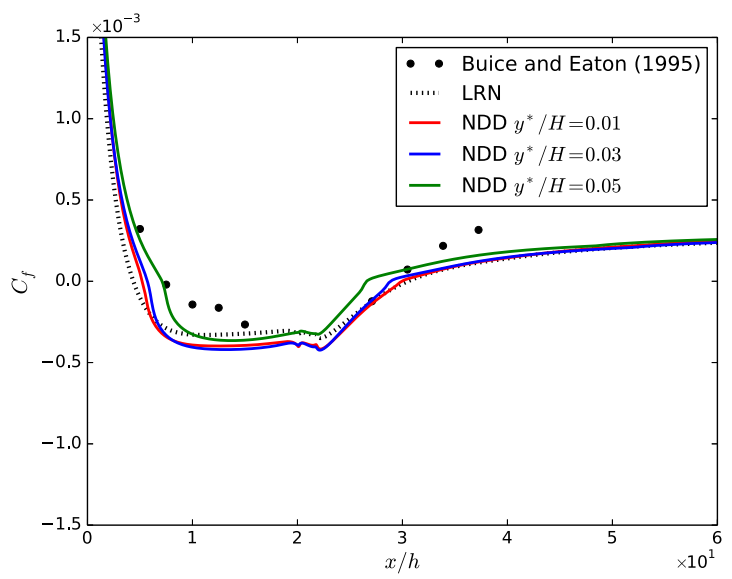

(a) Spalart-Allmaras

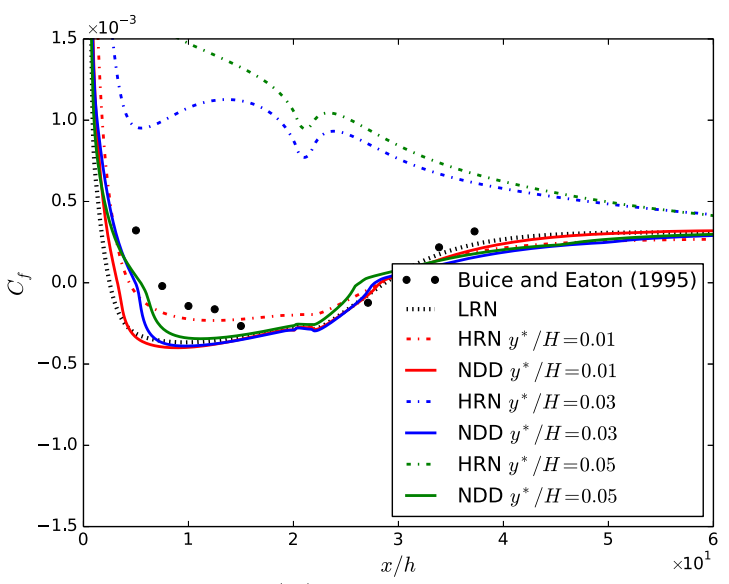

(b) $k-\omega \mathrm{SST}$

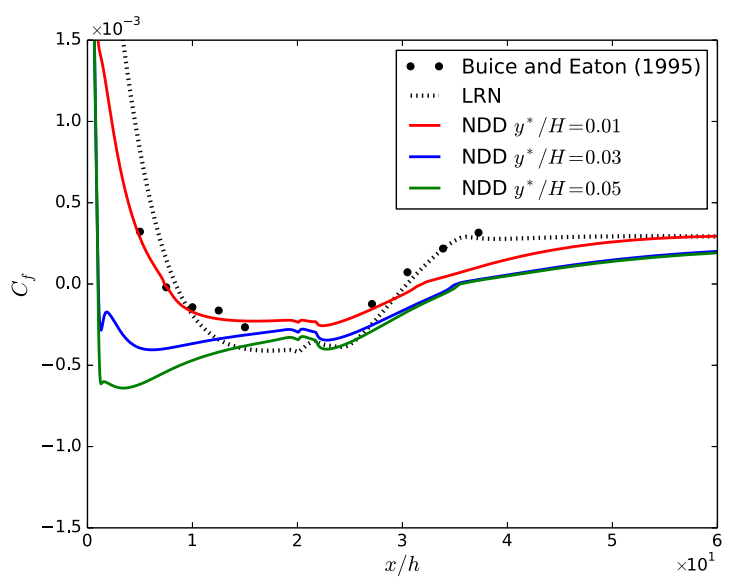

(c) $\mathrm{BL}-\overline{v^{2}} / k$

Fig. 8. $C_{f}$ along the inclined wall of the diffuser for with the three turbulence models for $y^{*} / H=0.01,0.03$ and 0.05 . 


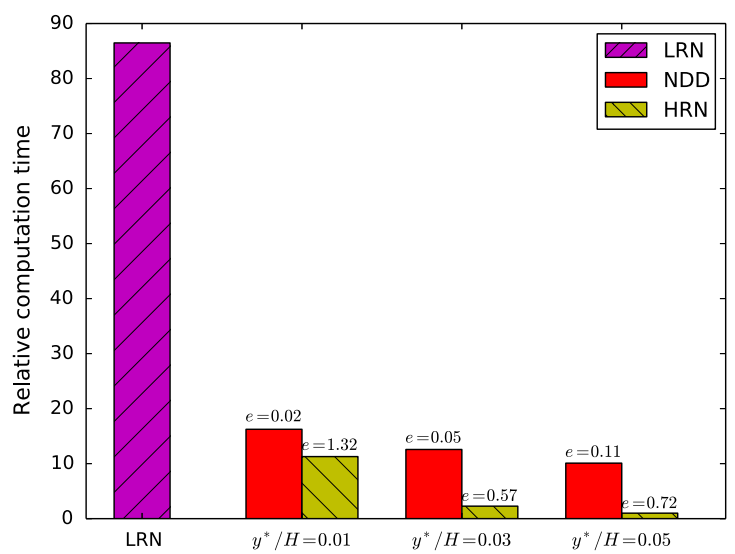

Fig. 9. Relative computation times of the diffuser simulations with the $k-\omega$ SST model. The times are normalised such that the fastest computation time is 1 . Also shown for each case is the maximum error in the skin friction factor between the LRN and NDD or HRN results.

In Eq. (48), $\Delta t$ is the time step, $\Omega_{i}$ is the volume of the $i$ th control volume and the sums run over the total number of cells $N$. The solution is considered converged when $D<\epsilon$ for every non-zero variable $\Phi$, where $\epsilon=10^{-6}$.

The total computation times for the diffuser simulations with the $k-\omega$ SST model are shown in Fig. 9. The times are normalised such that the fastest computation time is 1 . This occurs for the simulation with the HRN $k-\omega$ SST model with $y^{*} / H=0.05$. Also shown are the maximum errors in $C_{f}$ on the inclined wall, normalised by the wall shear stress of the channel at the inlet, $C_{f 0}$. The figures are calculated as

$e=\frac{\max \left(C_{f}^{\mathrm{LRN}}(x)-C_{f}^{\mathrm{NDD} \text { or } \mathrm{HRN}}(x)\right)}{C_{f 0}}$.

The LRN calculation takes roughly an order of magnitude longer than the NDD or HRN simulations. As $y^{*}$ increases the computation time increases with both HRN and NDD simulations. In the case of $y^{*} / H=0.01$ the NDD results are most similar to the LRN results yet the computation time is roughly one sixth.

Although the HRN simulations with $y^{*} / H=0.03$ and 0.05 are noticeably faster than the other simulations, Fig. $8 \mathrm{~b}$ indicates that no recirculation region is predicted in these cases. Therefore the accuracy of the solution is degraded. Moreover, these simulations will suffer from the largest mesh sensitivity, since the near-wall cell size is largest.

\section{Conclusions}

A non-overlapping NDD approach has been successfully implemented with the $k-\omega$ SST, Spalart-Allmaras and BL- $\overline{v^{2}} / k$ models for the first time. The NDD method has been shown to perform well enough on the cases of a plane channel flow and heated annulus flows at different Reynolds numbers. It has been found that the results obtained with IBCs show little sensitivity to the location of the interface boundary.

The NDD results on an asymmetric diffuser show that it is more accurate than conventional wall functions in flows with separation regions. The results are seen to converge to the LRN solution as $y^{*} \rightarrow 0$.

It has been shown that as the interface boundary is shifted into the core of the flow, the computation time decreases because a significantly coarser mesh can be used. Thus, it has been demonstrated that the NDD method with IBCs leads to a significantly shorter computation time without deteriorating the level of accuracy of the results. This makes the approach efficient for use in industrial applications.

\section{Acknowledgements}

This work was performed as part of a research project sponsored by EDF Energy. The first author is supported financially by the EPSRC (Grant No. EP/G037426/1) and EDF Energy R\&D UK Centre Limited. The authors are grateful to the anonymous referees for their useful remarks.

\section{Appendix A. Turbulence models used in this paper}

The governing equations for the three turbulence models used in this work are given below, along with the model constants.

The mean strain tensor $S_{i j}$ and the mean vorticity tensor $\Omega_{i j}$ are defined as

$S_{i j}=\frac{1}{2}\left(\partial_{j} U_{i}+\partial_{i} U_{j}\right)$

$\Omega_{i j}=\frac{1}{2}\left(\partial_{j} U_{i}-\partial_{i} U_{j}\right)$,

from which the following scalars are derived

$S=\sqrt{2 S_{i j} S_{i j}}$

$\Omega=\sqrt{2 \Omega_{i j} \Omega_{i j}}$.

The distance to the wall is denoted $d$.

\section{A.1. The Spalart-Allmaras model}

The Spalart-Allmaras model is a one-equation turbulence model that solves for a function $\tilde{v}$. The implementation used in this work differs from that in [32] in that it does not contain any terms involving the damping functions $f_{t 1}$ or $f_{t 2}$.

- The equation that $\tilde{v}$ obeys is

$$
\begin{aligned}
\frac{\partial(\rho \tilde{v})}{\partial t}+\nabla \cdot(\rho \tilde{v} \mathbf{U})= & c_{b 1} \rho \tilde{S} \tilde{v}-c_{w 1} \rho f_{w}\left(\frac{\tilde{v}}{d}\right)^{2}+\frac{c_{b 2} \rho}{\sigma} \nabla \tilde{v} \cdot \nabla \tilde{v} \\
& +\frac{1}{\sigma} \nabla \cdot((\mu+\rho \tilde{v}) \nabla \tilde{v}) .
\end{aligned}
$$

- The turbulent viscosity is calculated as

$$
\mu_{t}=\rho \tilde{v} f_{v 1} .
$$

- The damping functions are

$$
\begin{aligned}
& f_{v 1}=\frac{\chi^{3}}{\chi^{3}+c_{v 1}^{3}}, \\
& f_{v 2}=1-\frac{\chi}{1+\chi f_{v 1}},
\end{aligned}
$$

and

$f_{w}=g\left(\frac{1+c_{w 3}^{6}}{g^{6}+c_{w 3}^{6}}\right)^{1 / 6}$,

with the definitions

$$
\begin{aligned}
& \chi=\frac{\rho \tilde{v}}{\mu}, \\
& g=r+c_{w 2}\left(r^{6}-r\right),
\end{aligned}
$$


$r=\min \left(\frac{\tilde{v}}{\widetilde{S} \kappa^{2} d^{2}}, 10\right)$

$\bar{S}=\frac{\tilde{v}}{\kappa^{2} d^{2}} f_{v 2}$,

and

$\widetilde{S}= \begin{cases}\Omega+\bar{S} & \text { if } \bar{S} \geqslant-c_{2} \Omega, \\ \Omega+\frac{\Omega\left(c_{2}^{2} \Omega+c_{3} \bar{S}\right)}{\left(c_{3}-2 c_{2}\right) \Omega-\bar{S}} & \text { if } \bar{S}<-c_{2} \Omega .\end{cases}$

- The wall boundary condition is

$\tilde{v}_{w}=0$.

The model constants are given in Table A.3.

\section{A.2. The $k-\omega$ SST model}

The $k-\omega$ SST model [26] is a two-equation turbulence model.

- The $k$ equation is

$$
\frac{\partial(\rho k)}{\partial t}+\nabla \cdot(\rho k \mathbf{U})=\rho \mathcal{P}_{k}-\beta^{*} \rho k \omega+\nabla \cdot\left(\left(\mu+\frac{\mu_{t}}{\sigma_{k}}\right) \nabla k\right),
$$

and the $\omega$ equation is

$$
\begin{aligned}
& \frac{\partial(\rho \omega)}{\partial t}+\nabla \cdot(\rho \omega \mathbf{U})=\frac{\gamma}{\mu_{t}} \rho \mathcal{P}_{\omega}-\beta \rho \omega^{2} \\
& \quad+\nabla \cdot\left(\left(\mu+\frac{\mu_{t}}{\sigma_{\omega}}\right) \nabla \omega\right)+\frac{2 \rho}{\sigma_{\omega 2}}\left(1-F_{1}\right) \frac{1}{\omega} \nabla k \cdot \nabla \omega .
\end{aligned}
$$

- The turbulent viscosity is calculated as

$$
v_{t}=\frac{a_{1} k}{\max \left(a_{1} \omega, S F_{2}\right)} \text {. }
$$

- The model functions are

$$
\begin{aligned}
& \mathcal{P}_{k}=\min \left(-\overline{u_{i} u_{j}} \frac{\partial U_{i}}{\partial x_{j}}, c_{1} \beta^{*} k \omega\right), \\
& \mathcal{P}_{\omega}=-\overline{u_{i} u_{j}} \frac{\partial U_{i}}{\partial x_{j}}, \\
& -\overline{u_{i} u_{j}}=2 \mu_{t} S_{i j}-\frac{2}{3} k \delta_{i j}, \\
& F_{1}=\tanh \left(\varphi_{1}^{4}\right), \\
& F_{2}=\tanh \left(\varphi_{2}^{2}\right), \\
& \varphi_{1}=\min \left(\max \left(\frac{k^{1 / 2}}{\beta^{*} \omega y}, \frac{500 \mu}{y^{2} \rho \omega}\right), \frac{4 \rho k}{\sigma_{\omega 2} \mathrm{CD}_{k \omega} y^{2}}\right), \\
& \mathrm{CD}_{k \omega}=\max \left(\frac{2 \rho}{\sigma_{\omega 2} \omega} \nabla k \cdot \nabla \omega, 10^{-20}\right),
\end{aligned}
$$

and

$$
\varphi_{2}=\max \left(\frac{2 k^{1 / 2}}{\beta^{*} \omega y}, \frac{500 \mu}{\rho \omega y^{2}}\right)
$$

- Every model constant $c$ is calculated by blending two constants $c_{1}$ and $c_{2}$ with the expression

$c=c_{1} F_{1}+c_{2}\left(1-F_{1}\right)$,

The model constants are given in Table A.4. The constants $\gamma_{i}$ with $i=1,2$ are

$\gamma_{i}=\frac{\beta_{i}}{\beta^{*}}-\frac{\kappa^{2}}{\sqrt{\beta^{*}} \sigma_{\omega i}}$

- The wall boundary condition for $k$ is

$k_{w}=0$.

The required asymptotic behaviour of $\omega$ near a wall at $y=0$ is $\omega(y) \sim y^{-2}$ as $y \rightarrow 0$. A common boundary condition is [25]

$\omega_{w}=\frac{60 \mu}{\beta_{1} \rho d_{1}^{2}}$

where $d_{1}$ is the distance from the wall to the near-wall cell centre.

\section{A.3. The $\mathrm{BL}-\overline{v^{2}} / k$ model}

The BL- $\overline{v^{2}} / k$ model [4] is a four equation turbulence model that solves for the functions $k, \varepsilon_{h}, \varphi$ and $\alpha$. It is based on the $\overline{v^{2}}-f$ model of Durbin [15]. The turbulent kinetic energy $k$ is analogous to that found in many $k-\varepsilon$ equations, however the dissipation differs by the change of variables

$\varepsilon_{h}=\varepsilon-\frac{k}{\varepsilon} E-\frac{1}{2} v \nabla^{2} k$,

where $E$ is the so-called $E$-term. This substitution is the origin of the factors of $\frac{1}{2}$ in the viscous terms of the governing equations. The function $\varphi=\overline{v^{2}} / k$, introduced in Laurence et al. [24], is an estimation of the wall-normal turbulent fluctuations and is non-dimensional. Non-local effects are captured by the function $\alpha$, which obeys an elliptic equation and is also non-dimensional.

- The $k$ equation is

$$
\frac{\partial(\rho k)}{\partial t}+\nabla \cdot(\rho k \mathbf{U})=\rho \mathcal{P}-\rho \varepsilon_{h}-\rho E+\nabla \cdot\left(\left(\frac{\mu}{2}+\frac{\mu_{t}}{\sigma_{k}}\right) \nabla k\right),
$$

the $\varepsilon_{h}$ equation is

$$
\frac{\partial\left(\rho \varepsilon_{h}\right)}{\partial t}+\nabla \cdot\left(\rho \varepsilon_{h} \mathbf{U}\right)=\frac{C_{\varepsilon 1} \rho \mathcal{P}-C_{\varepsilon 2}^{*} \rho \varepsilon_{h}}{T}+\nabla \cdot\left(\left(\frac{\mu}{2}+\frac{\mu_{t}}{\sigma_{\varepsilon_{h}}}\right) \nabla \varepsilon_{h}\right),
$$

the $\varphi$ equation is

$$
\begin{aligned}
\frac{\partial(\rho \varphi)}{\partial t}+\nabla \cdot(\rho \varphi \mathbf{U})= & \rho\left(1-\alpha^{3}\right) f_{w}+\rho \alpha^{3} f_{h}-\rho \mathcal{P} \frac{\varphi}{k} \\
& +\frac{2 \mu_{t}}{\sigma_{k} k} \nabla \varphi \cdot \nabla k+\nabla \cdot\left(\left(\frac{\mu}{2}+\frac{\mu_{t}}{\sigma_{\varphi}}\right) \nabla \varphi\right),
\end{aligned}
$$

Table A.3

The constants for the Spalart-Allmaras model.

\begin{tabular}{llllllllll}
\hline$\sigma$ & $\kappa$ & $c_{b 1}$ & $c_{b 2}$ & $c_{w 1}$ & $c_{w 2}$ & $c_{w 3}$ & $c_{v 1}$ & $c_{2}$ & $c_{3}$ \\
\hline$\frac{2}{3}$ & 0.42 & 0.1355 & 0.622 & $\frac{c_{b 1}}{\kappa^{2}}+\frac{1+c_{b 2}}{\sigma}$ & 0.3 & 2 & 7.1 & 0.7 & 0.9 \\
\hline
\end{tabular}

Table A.4

The constants for the $k-\omega$ SST model.

\begin{tabular}{clllllllll}
\hline$\sigma_{k 1}$ & $\sigma_{k 2}$ & $\sigma_{\omega 1}$ & $\sigma_{\omega 2}$ & $\beta_{1}$ & $\beta_{2}$ & $a_{1}$ & $c_{1}$ & $\kappa$ & $\beta^{*}$ \\
\hline$\frac{1}{0.85}$ & 1 & 2 & $\frac{1}{0.856}$ & 0.075 & 0.0828 & 0.31 & 10 & 0.42 & 0.09 \\
\hline
\end{tabular}


Table A.5

The constants for the BL- $\overline{v^{2}} / k$ model.

\begin{tabular}{lllllllllllll}
\hline$\sigma_{k}$ & $\sigma_{\varepsilon_{h}}$ & $\sigma_{\varphi}$ & $C_{\mu}$ & $C_{k}$ & $C_{\eta}$ & $C_{1}$ & $C_{2}$ & $C_{T}$ & $C_{L}$ & $C_{\varepsilon 1}$ & $C_{\varepsilon 2}$ & $C_{\varepsilon 3}$ \\
\hline 1 & 1.5 & 1 & 0.22 & 2.3 & 75 & 1.7 & 0.9 & 4 & 0.164 & 1.44 & 1.83 & 0.4 \\
\hline
\end{tabular}

and $\alpha$ obeys the equation

$\alpha-L^{2} \nabla^{2} \alpha=1$.

- The turbulent viscosity is calculated as

$\mu_{t}=\rho c_{\mu} \varphi k \min \left(T, T_{\text {lim }}\right)$.

- The model functions are

$\rho \mathcal{P}=\frac{1}{2} \mu_{t} S^{2}$

$E=2 C_{k}(1-\alpha)^{3} \frac{k}{\varepsilon_{h}} \frac{\mu \mu_{t}}{\rho^{2}}\left(\frac{\partial^{2} U_{i}}{\partial x_{k} \partial x_{j}}\right)\left(\frac{\partial^{2} U_{i}}{\partial x_{k} \partial x_{j}}\right)$

$f_{w}=-\frac{\varepsilon_{h} \varphi}{2 k}$

$f_{h}=-\frac{1}{T}\left(C_{1}-1+C_{2} \frac{\mathcal{P}}{\varepsilon_{h}}\right)\left(\varphi-\frac{2}{3}\right)$,

$L=\sqrt{C_{L}^{2}\left(\frac{k^{3}}{\varepsilon_{h}^{2}}+C_{\eta}^{2} \frac{\mu^{3 / 2}}{\rho^{3 / 2} \varepsilon_{h}^{1 / 2}}\right)}$

and

$T=\sqrt{\frac{k^{2}}{\varepsilon_{h}^{2}}+C_{T}^{2} \frac{\mu}{\rho \varepsilon_{h}}}$

with

$T_{\text {lim }}=\frac{0.6}{\sqrt{3} c_{\mu} \varphi S}$

- The model constants are given in Table A.5. However $C_{\varepsilon 2}^{*}$ is calculated as

$$
\begin{aligned}
C_{\varepsilon 2}^{*}= & C_{\varepsilon 2} \\
& +\alpha^{3}\left(C_{\varepsilon 3}-C_{\varepsilon 2}\right) \tanh \left(\left|\frac{1}{\varepsilon_{h}} \frac{\partial}{\partial x_{j}}\left(\frac{\mu_{t}}{\rho \sigma_{k}} \frac{\partial k}{\partial x_{j}}\right)\right|^{3 / 2}\right) .
\end{aligned}
$$

- The boundary conditions at the wall are

$$
\begin{aligned}
& k_{w}=0, \\
& \left.\varepsilon_{h}\right|_{w}=\frac{\mu k_{1}}{\rho d_{1}^{2}}, \\
& \varphi_{w}=0, \\
& \alpha_{w}=0,
\end{aligned}
$$

where $k_{1}$ and $d_{1}$ are respectively the turbulent kinetic energy the distance from the wall at the near-wall cell centre.

\section{References}

[1] Aleksin V, Utyuzhnikov S. Implementation of near-wall boundary conditions for modeling boundary layers with free-stream turbulence. Appl Math Model 2014;38(14):3591-606. <http://www.sciencedirect.com/science/article/pii/ S0307904X1300807X>.
[2] Allmaras SR, Johnson FT, Spalart PR. Modifications and clarifications for the implementation of the Spalart-Allmaras turbulence model. In: 7th international conference on computational fluid dynamics. ICCFD7-1902. Big Island, Hawaii; July 2012.

[3] Archambeau F, Namane M, Sakiz M. Code Saturne: a finite volume code for turbulent flows. Int. J. Finite Vol 2004;1.

[4] Billard F, Laurence D. A robust $k-\varepsilon-\overline{v^{2}} / k$ elliptic blending turbulence model applied to near-wall, separated and buoyant flows. Int J Heat Fluid Flow 2012;33(1):4558. <http://www.sciencedirect.com/science/article/pii/ S0142727X11001512>.

[5] Billard F, Laurence D, Osman K. Adaptive wall functions for an elliptic blending eddy viscosity model applicable to any mesh topology. Flow Turbul Combust 2015:1-26.

[6] Billard F, Osman K, Laurence D. Development of adaptive wall functions for an elliptic-blending model. In: 7th symposium on turbulence, heat and mass transfer. Palermo, Italy; 2012.

[7] Buice CU, Eaton JK. Experimental investigation of flow through an asymmetric plane diffuser. Centre Turbul Res Ann Res Briefs 1995;1995:117-20.

[8] Cabot W, Moin P. Approximate wall boundary conditions in the large-eddy simulation of high Reynolds number flow. Flow Turbul Combust 1999;63:269-91.

[9] Chung SY, Rhee GH, Sung HJ. Direct numerical simulation of turbulent concentric annular pipe flow: Part 1: flow field. Int J Heat Fluid Flow 2002;23(4):426-40. <http://www.sciencedirect.com/science/article/pii/ S0142727X02001406>

[10] Chung SY, Sung HJ. Direct numerical simulation of turbulent concentric annular pipe flow: Part 2: heat transfer. Int $\mathrm{J}$ Heat Fluid Flow 2003;24(3):399-411. <http://www.sciencedirect.com/science/article/pii/ S0142727X03000171>

[11] Craft TJ, Gant SE, Iacovides H, Launder BE. A new wall function strategy for complex turbulent flows. Numer Heat Transf Part B: Fundam 2004;45(4):301-18. <http://www.tandfonline.com/doi/abs/10.1080/ $10407790490277931>$

[12] Craft TJ, Gerasimov AV, Iacovides H, Launder BE. Progress in the generalization of wall-function treatments. Int J Heat Fluid Flow 2002;23(2):148-60. <http:// www.sciencedirect.com/science/article/pii/S0142727X01001436>.

[13] Davis ES. Heat transfer and pressure drop in annuli. Trans Am Soc Mech Eng 1943;65:755-60.

[14] Duprat C, Balarac G, Metais O, Congedo PM, Brugiere O. A wall-layer model for large-eddy simulations of turbulent flows with/out pressure gradient. Phys Fluids 2011;23(1):015101. <http://link.aip.org/link/?PHF/23/015101/1>.

[15] Durbin PA. Near-wall turbulence closure modeling without 'damping functions'. Theoret Comput Fluid Dyn 1991;3:1-13.

[16] Durbin PA. Limiters and wall treatments in applied turbulence modeling. Fluid Dyn Res 2009;41(1):012203.

[17] Gant SE. Development and application of a new wall function for complex turbulent flows. Ph.D. thesis. Manchester: Department of Mechanical, Aerospace \& Manufacturing Engineering, UMIST; 2002.

[18] Grotjans H, Menter FR. Wall functions for general application CFD codes. In: Papailou, editor. ECCOMAS 98. European congress on computational methods in applied sciences and engineering. Swansea; 1998. p. 1112-7.

[19] Hellsten A, Rautaheimo P, editors. 8th ERCOFTAC/IAHR/COST workshop on refined turbulence modelling. ERCOFTAC. Finland: Helsinki University of Technology, Espoo; June 1999.

[20] Kalitzin G, Medic G, Iaccarino G, Durbin P. Near-wall behavior of RANS turbulence models and implications for wall functions. J Comput Phys 2005;204(1):265-91. <http://www.sciencedirect.com/science/article/pii/ S0021999104004164>.

[21] Kaltenbach H-J, Fatica M, Mittal R, Lund T, Moin P. Study of flow in a planar asymmetric diffuser using large-eddy simulation. J Fluid Mech 1999;390(1):151-85.

[22] Kays W, Leung E. Heat transfer in annular passages-hydrodynamically developed turbulent flow with arbitrarily prescribed heat flux. Int J Heat Mass Transfer 1963;6(7):537-57. <http://www.sciencedirect.com/science/ article/pii/0017931063900127>.

[23] Knopp T, Alrutz T, Schwamborn D. A grid and flow adaptive wall-function method for RANS turbulence modelling. J Comput Phys 2006;220(1):19-40.

[24] Laurence DR, Uribe JC, Utyuzhnikov SV. A robust formulation of the $\overline{v^{2}}-f$ model. Flow Turbul Combust 2004;73:169-85. <http://cfd.mace.manchester. ac.uk/coffee/papers/JU-DL.pdf>.

[25] Menter FR. Two-equation eddy-viscosity turbulence models for engineering applications. AIAA J 1994:32(8):1598-605.

[26] Menter FR, Kuntz M, Langtry R. Ten years of industrial experience with the SST turbulence model. Turbul Heat Mass Transfer 2003;4:625-32.

[27] Moser RD, Kim J, Mansour NN. Direct numerical simulation of turbulent channel flow up to $R e_{\tau}=590$. Phys Fluids 1999;11(4):943-5.

[28] Nallasamy M. Turbulence models and their applications to the prediction of internal flows: a review. Comput Fluids 1987;15(2):151-94. <http:// www.sciencedirect.com/science/article/pii/S0045793087800038>.

[29] Ould-Rouiss M, Redjem-Saad L, Lauriat G. Direct numerical simulation of turbulent heat transfer in annuli: effect of heat flux ratio. Int J Heat Fluid Flow 2009;30(4):579-89.

[30] Popovac M, Hanjalić K. Compound wall treatment for RANS computation of complex turbulent flows and heat transfer. Flow Turbul Combust 2007;78(2):177-202. <http://www.ingentaconnect.com/content/klu/appl/ 2007/00000078/00000002/00009067>. 
[31] Rodi W, Scheuerer G. Scrutinizing the k-epsilon turbulence model under adverse pressure gradient conditions. ASME Trans J Fluids Eng 1986;108: 174-9.

[32] Spalart PR, Allmaras SR. A one-equation turbulence model for aerodynamic flows. La Recherche Aérospatiale 1994(1):5-21.

[33] Utyuzhnikov SV. Generalized wall functions and their application for simulation of turbulent flows. Int J Numer Meth Fluids 2005;47(1011):1323-8. <http://dx.doi.org/10.1002/fld.873>.

[34] Utyuzhnikov SV. The method of boundary condition transfer in application to modeling near-wall turbulent flows. Comput Fluids 2006;35(10):1193-204. <http://www.sciencedirect.com/science/article/pii/S0045793005000836>.

[35] Utyuzhnikov SV. Robin-type wall functions and their numerical implementation. Appl Numer Math 2008;58(10):1521-33. <http:// www.sciencedirect.com/science/article/pii/S0168927407001432>.
[36] Utyuzhnikov SV. Domain decomposition for near-wall turbulent flows Comput Fluids 2009;38(9):1710-7. <http://www.sciencedirect.com/science/ article/pii/S0045793009000371>.

[37] Utyuzhnikov SV. Interface boundary conditions in near-wall turbulence modeling. Comput Fluids 2012;68:186-91. <http:/l www.sciencedirect.com/science/article/pii/S0045793012002915>.

[38] van Driest ER. On turbulent flow near a wall. J Aerosp Sci 1956;23:1007-11.

[39] Watson MAP. The performance of a square rib type of heat transfer surface. Tech rep. RD/B/N1738. Central Electricity Generating Board; December 1970.

[40] Yu B, Kawaguchi Y, Kaneda M, Ozoe H, Churchill SW. The computed characteristics of turbulent flow and convection in concentric circular annuli. Part II. Uniform heating on the inner surface. Int J Heat Mass Transfer 2005;48(34):621-34. 